\title{
Loss of $N K X 3-1$ as a potential marker for an increased risk of occult lymph node metastasis and poor prognosis in oral squamous cell carcinoma
}

\author{
KEN MIYAGUCHI $^{1}$, NARIKAZU UZAWA ${ }^{2}$, KAORU MOGUSHI ${ }^{3}$, KEN-ICHIRO TAKAHASHI $^{2}$, \\ CHIEKO MICHIKAWA ${ }^{2}$, YOSHIMI NAKATA ${ }^{2}$, JUN SUMINO ${ }^{2}$, NORIHIKO OKADA ${ }^{4}$, \\ HIROSHI MIZUSHIMA ${ }^{5}$, YUTAKA FUKUOKA ${ }^{6}$ and HIROSHI TANAKA ${ }^{3}$
}

\begin{abstract}
Departments of ${ }^{1}$ Bioinformatics and ${ }^{2}$ Maxillofacial Surgery, Graduate School; ${ }^{3}$ Department of Computational Biology, Graduate School of Biomedical Science; ${ }^{4}$ Department of Diagnostic Oral Pathology, Graduate School, Tokyo Medical and Dental University; ${ }^{5}$ Center for Public Health Informatics, National Institute of Public Health; ${ }^{6}$ Department of Biosystem Modeling, Graduate School of Biomedical Science, Tokyo Medical and Dental University, Tokyo, Japan
\end{abstract}

Received November 12, 2011; Accepted December 19, 2011

DOI: $10.3892 /$ ijo.2012.1373

\begin{abstract}
The prognosis of oral squamous cell carcinoma (OSCC) is significantly dependent on the existence of cervical lymph node metastasis (LNM), with the overall survival rate being much lower in patients with LNM. Primary causes and molecular mechanisms of LNM are still largely unclear. We hypothesized that factors related with cancer progress and/or prognosis in OSCC are revealed by genome-wide investigation of DNA copy number aberrations (CNAs). In order to find biomarkers for occult LNM of OSCC, we comprehensively investigated genomic DNAs from 60 OSCC patients using Affymetrix mapping arrays and statistically analyzed correlations between CNAs of genes and the presence of occult LNM in the patients. The genome-wide CNA study indicated significant correlations between the presence of occult LNM and CNAs of certain genes. Through a literature survey, we narrowed down the candidates and focused on loss of NKX3-1, which is a homeodomain-containing transcription factor. $N K X 3-1$ is known as a tumor suppressor gene in prostate cancer but has never been reported in OSCC. Quantitative RT-PCR and immunohistochemistry (IHC) analyses also showed significantly lower expression of NKX3-1 in the cases with occult LNM, which was further validated by IHC analysis in independent cases. The survival analyses indicated that
\end{abstract}

Correspondence to: Dr Hiroshi Mizushima, Center for Public Health Informatics, National Institute of Public Health, 2-3-6 Minami, Wako-shi, Saitama 351-0197, Japan

E-mail: hmizushi@niph.go.jp

Dr Yutaka Fukuoka, Department of Biosystem Modeling, Graduate School of Biomedical Science, Tokyo Medical and Dental University, 1-5-45 Yushima, Bunkyo-ku, Tokyo 113-8510, Japan

E-mail: yu_fukuoka@ieee.org

Key words: oral squamous cell carcinoma, occult lymph node metastasis, DNA copy number, SNP array, NKX3-1
$N K X 3-1$ loss is a significant risk factor to decrease the diseasefree survival (DFS) and the overall survival (OS) rates. This is the first time that the significant association of $N K X 3-1$ loss and occult LNM was indicated in OSCC. The present results suggest that loss of $N K X 3-1$ may be a potential biomarker for occult LNM of OSCC.

\section{Introduction}

Oral squamous cell carcinoma (OSCC) is the most common head and neck carcinoma, accounting for more than 260,000 cases worldwide each year (1). Although therapies for OSCC have recently been improved, only slight progress has been observed in the mortality rates over the last two decades (2). The most important prognostic factor in OSCC is the presence of lymph node metastasis (LNM). In fact, the 5-year overall survival rate in patients with LNM is approximately $25 \%$ lower than in patients without LNM (3). Especially, nodal metastasis diagnosed after surgical resection of a primary tumor site is more severe for survival and known as occult LNM even when the patient is diagnosed without cervical LNM (N0 neck) at the first visit. Therefore, the appropriate management of occult LNM is required and currently two major approaches are applied to manage the NO neck in OSCCs; an elective neck dissection of cervical lymph node(s) and a 'wait-and-see' policy $(4,5)$. In a case of stage N0 neck with a risk of occult LNM greater than $20 \%$, neck dissection is a standard procedure for elective treatment (6). The risk of metastatic potential can vary according to the site, size, histopathological and genetic features of a primary tumor. Much controversy exists over the decision of the treatment with or without a preventive lymphadenectomy based on accurate risk stratification of potential occult LNM.

Although the associations between metastasis and particular genomic aberrations have been reported $(7,8)$, the molecular mechanism of LNM is still not fully understood. In addition to uncovering how OSCC cells spread and migrate from the primary site to cervical lymph nodes, it is also valua- 
ble to identify genomic aberrations that are associated with LNM and are thus potential biomarkers to increase the accuracy of the prediction of occult LNM. A genome-wide copy number $(\mathrm{CN})$ analysis by a high-density microarray allows us to identify numerous CNAs of genes, in which some of them may have important influence to tumor progression and poor prognosis in malignancy $(9,10)$. We anticipate that factors related with occult LNM are also revealed by a comprehensive study of DNA copy number aberrations (CNAs) in OSCC. However, to the best of our knowledge, there is no genomewide CNA study reporting genes associated with occult LNM of OSCC although gene- or chromosomal site-specific analyses have been reported $(11,12)$.

The purpose of this study was to identify significant markers associated with occult LNM by a genome-wide CN analysis of the primary tumor sites. A high density SNP array was applied to screen occult LNM-related aberrations of genes in 60 OSCC patients.

\section{Materials and methods}

Patients and tissue samples. Fresh-frozen tissue samples were collected from 60 OSCC patients (39 male, 21 female) who had undergone surgical resection at the Department of Maxillofacial Surgery, Graduate School, Tokyo Medical and Dental University (TMDU; Tokyo, Japan) between 1999 and 2007. None of these patients received preoperative treatment. This study was approved by the institutional review board. Informed consent was obtained from all patients in accordance with the guidelines by the review board. The cancers were located in the tongue (34 samples), mandibular gingiva (14 samples), floor of the mouth (4 samples), buccal mucosa (6 samples), maxilla gingiva (1 sample), and pterygopalatine fossa (1 sample). Parts of the tumor specimens were collected in cryotubes, frozen just after the resection and stored at $-80^{\circ} \mathrm{C}$ until used in the following analyses. The remaining specimens were prepared as formalin-fixed, paraffin-embedded (FFPE) samples and used for immunohistochemistry (IHC) assay. As an independent sample set for the IHC assay, FFPE sections were prepared from 12 additional OSCC patients (6 with occult LNM, 6 without occult LNM) who underwent surgical therapy at TMDU dental hospital between 1999 and 2009.

Genomic DNA extraction from primary tumors. Genomic DNAs were extracted from the frozen tumor specimens and isolated using a QIAamp DNA Mini kit (Qiagen, Valencia, CA). Contaminated RNAs were eliminated using RNase A during the purification process. The quantities and qualities of purified genomic DNAs were assessed by NanoDrop (NanoDrop Technologies, Wilmington, DE) and gel electrophoresis.

Genome-wide copy number analysis. The experiment was performed by strictly following the assay manual and using a GeneChip human mapping 250K Sty array (Affymetrix, Santa Clara, CA), which contained 238,229 SNP probe sets. The concentration of starting materials was standardized by diluting genomic DNA samples to $50 \mathrm{ng} / \mu 1$ (250 ng of genomic DNA) with reduced EDTA TE buffer. A Sty I assay was performed in this study, by applying Sty I digestion of genomic DNA and ligation with adaptor Sty I prior to the PCR reaction. Amplicons were fragmented after the purification, followed by a labeling reaction. After $16 \mathrm{~h}$ of hybridization at $49^{\circ} \mathrm{C}$, the microarrays were transferred to a fully automated system (GeneChip Fluidic Station 450; Affymetrix) for the washing and staining steps. After the staining with streptavidin phycoerythrin (SAPE), images of the microarrays were scanned with an Affymetrix laser scanner 3000-7G. The raw intensity data of SNP probe sets were first analyzed by Affymetrix genotyping console 4.0 software to produce the genotype data. The CNA data were generated by the estimation of the copy number for each SNP probe set by comparing each normalized intensity data with Affymetrix SNP array data set of 50 normal Asians (Japanese and Chinese) from the HapMap database (http:// www.hapmap.org/). The microarray data for the 60 samples used in this study are available from the NCBI Gene Expression Omnibus (http://www.ncbi.nlm.nih.gov/geo/) under accession number GSE31984.

Comprehensive correlational analysis between CNAs of genes and occult LNM. To identify an individual copy number for each gene, we first determined segmented CNA regions based on integrated chromosomal positions of SNP probes which had a unique $\mathrm{CN}$ profile; loss/deletion, neutral or gain/ amplification. The CNA regions were annotated with relative gene information which included 22,658 gene information downloaded from the EntrezGene database (http://www. ncbi.nlm.nih.gov/sites/entrez?db=gene). The Fisher's exact test with the Bonferroni correction was applied on the gene CNA data from the 60 samples combined with the presence of occult LNM to determine the occult LNM-associated genes with $\mathrm{p}<0.05$.

Quantitative analysis by real-time RT-PCR. Using RNeasy Mini Kit (Qiagen, Valencia, CA), total RNAs were extracted from 19 OSCC tissues (9 cases without occult LNM and 10 cases with occult LNM) that were from the 60 samples used for the CNA analysis and reverse transcribed to cDNA using high-capacity cDNA reverse transcription kits (Applied Biosystems, Foster City, CA). Real-time RT-PCR was applied to quantitatively confirm the effect of $N K X 3-1$ loss by comparing the samples of patients with and without occult LNM. The sequences of PCR primer sets were forward primer: 5'-CAGTCCCTACTGAGTACTCTTTCTCTC-3', reverse primer: 5'-CACAGTGAAATGTGTAATCCTTGC-3' for $N K X 3-1$ and forward primer: 5'-CTGGAACGGTGAAG GTGACA-3', reverse primer: 5'-AAGGGACTTCCTGTAA CAACGCA-3' for $A C T B$ as endogenous control. Expression level of vascular endothelial growth factor $\mathrm{C}(V E G F-C)$ was also determined to compare with the relative quantities of $N K X 3-1$ in the same cDNA sample set because $N K X 3-1$ is known to regulate expression of $V E G F-C$ (13). The forward and reverse PCR primers were 5'-CACGAGCTACCTCAGCAAGA-3' and 5'-GCTGCCTGACACTGTGGTA-3', respectively. The PCR reaction was carried out with PowerSYBR Green PCR Master Mix (Applied Biosystems). The signals of the samples were detected by the 7500 Fast Real-Time PCR System (Applied Biosystems) with 7500 Fast System software v1.4.0, and the 


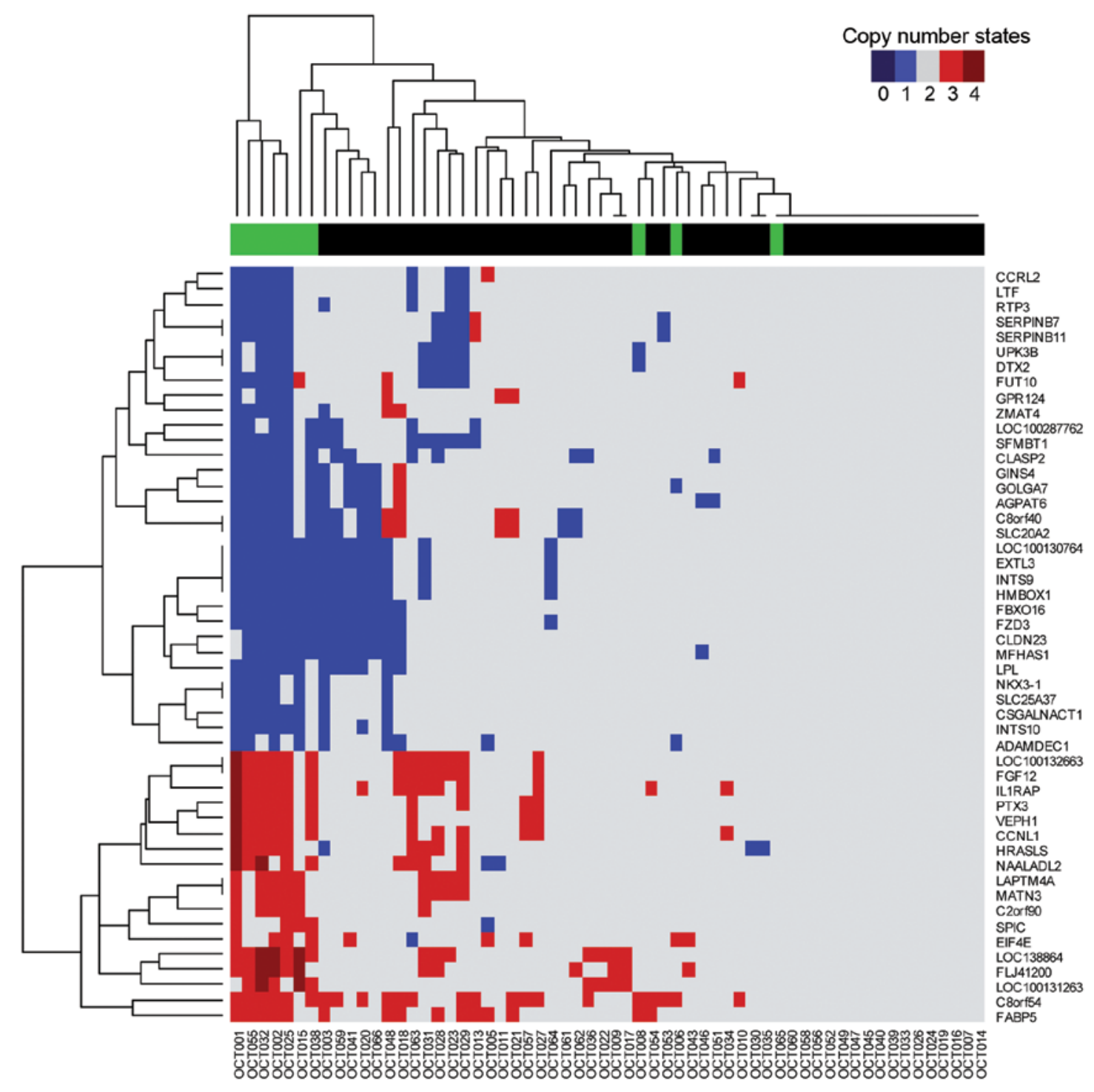

Figure 1. A heatmap with hierarchical clustering for a correlation between CNA profile of top 50 genes with lower p-values and the presence of occult LNM based on Mapping array data for 60 OSCC samples. Affymetrix copy number states are represented by colors; dark blue for 0 (deletion), blue for 1 (loss), gray for 2 (neutral), red for 3 (gain), and dark red for 4 (amplification). The presence of occult LNM is shown by a green bar at the top of the heatmap.

relative quantities of samples were calculated with an $\Delta \Delta \mathrm{C}_{t}$ method with the software and DataAssist v3.0 (Applied Biosystems). Each assay was performed in triplicate.

Immunohistochemistry assay for NKX3-1. Immunohistochemical staining was performed with a monoclonal antibody for NKX3-1 protein (1:200; abcam, Cambridge, UK) on the FFPE OSCC tissue specimens, which were 7 samples with occult LNM and 8 samples without occult LNM in the original sample set and 6 samples with occult LNM and 6 samples without occult LNM in the independent sample set. First, representative sections were stained with hematoxylin and eosin (H\&E) and histologically evaluated by a pathologist. The sections were deparaffinized by xylene, transferred to graded ethanol, and rehydrated in running water. Then, after antigen retrieval by autoclave treatment $\left(121^{\circ} \mathrm{C}, 40 \mathrm{~min}\right)$ in citrate buffer ( $\mathrm{pH}$ 6.0, $0.01 \mathrm{M})$, the sections were quenched for endogenous peroxidase activity and blocked with a blocking reagent of a Histofine SAB-PO kit (Nichirei, Tokyo, Japan). Anti-NKX3-1 antibody at a dilution of 1:200 was applied on the sections and incubated at room temperature for $30 \mathrm{~min}$, followed by overnight incubation at $4^{\circ} \mathrm{C}$. Subsequently, specimens were treated by the labeled streptavidin-biotinylated complex (LSAB) method using reagents of Histofine SAB-PO kit following the manufacturer protocol and stained by a 3,3'-diaminobenzidine (DAB; Merck, Darmstadt, Germany) solution containing hydrogen peroxide for $5 \mathrm{~min}$. The sections were then thoroughly counterstained with hematoxylin. As negative controls, tissues were stained with PBS instead of the primary antibody solution. For each of the 27 tissue specimens in the original and independent sample sets, the numbers of positively stained carcinoma cells and non-stained carcinoma cells were separately counted in 5,000 $\mu \mathrm{m}^{2}$ and the counting was repeated for nine additional areas under a light microscope. The ratio of positively stained cells over the total number of cells in a certain area and average of the ratio from the ten areas were calculated. 
Table I. Clinical characteristics of 60 OSCC cases and correlation with $N K X 3-1$ loss.

\begin{tabular}{|c|c|c|c|}
\hline \multirow{2}{*}{$\begin{array}{l}\text { Clinicopathological } \\
\text { parameters }\end{array}$} & \multicolumn{2}{|c|}{$\mathrm{CN}$ of $N K X 3-1$} & \multirow[b]{2}{*}{ p-value ${ }^{a}$} \\
\hline & Normal & Loss & \\
\hline Age & & & 0.436 \\
\hline$<60$ & 21 & 4 & \\
\hline$\geq 60$ & 32 & 3 & \\
\hline Gender & & & 1.000 \\
\hline Male & 34 & 5 & \\
\hline Female & 19 & 2 & \\
\hline Disease site & & & 0.180 \\
\hline Tongue & 31 & 3 & \\
\hline Lower gingiva & 11 & 3 & \\
\hline Upper gingiva & 0 & 1 & \\
\hline Buccal mucosa & 6 & 0 & \\
\hline Floor of mouth & 4 & 0 & \\
\hline Pterygopalatine fossa & 1 & 0 & \\
\hline Growth pattern & & & 0.741 \\
\hline Superficial/exophitic & 25 & 4 & \\
\hline Endophitic & 22 & 2 & \\
\hline Undetermined & 6 & 1 & \\
\hline Cellular differentiation & & & 0.580 \\
\hline Well/Moderate & 44 & 7 & \\
\hline Poor & 9 & 0 & \\
\hline Pathological T stage & & & 1.000 \\
\hline I, II & 40 & 6 & \\
\hline III, IV & 13 & 1 & \\
\hline Mode of invasion & & & 0.645 \\
\hline $1-3$ & 23 & 5 & \\
\hline $4 C, 4 D$ & 13 & 1 & \\
\hline Undetermined & 17 & 1 & \\
\hline Recurrence $^{\mathrm{b}}$ & & & 0.012 \\
\hline Negative & 42 & 2 & \\
\hline Positive & 11 & 5 & \\
\hline Occult lymph node metastasis & & & $<0.001$ \\
\hline Negative & 48 & 2 & \\
\hline Positive & 5 & 5 & \\
\hline
\end{tabular}

${ }^{\text {ap}} \mathrm{P}$-values were calculated by the Fisher's exact test; ${ }^{\text {b}}$ Recurrence includes primary recurrence, regional recurrence and distant metastasis.

Metastasis-free and overall survival analyses. To identify potential correlations between the survival rate and CNAs, we applied the Kaplan-Meier method for analyzing the diseasefree survival (DFS) and the overall survival (OS) in two groups of the 60 OSCC patients: one with loss of $N K X 3-1$ and the other without CNA in NKX3-1. Follow-up periods for DFS and OS were calculated based on the interval between the first visit and the date of the patient's death or the last follow-up.

Statistical analysis. The statistical analyses were performed with the statistical software R 2.11.1 (http://www.r-project.

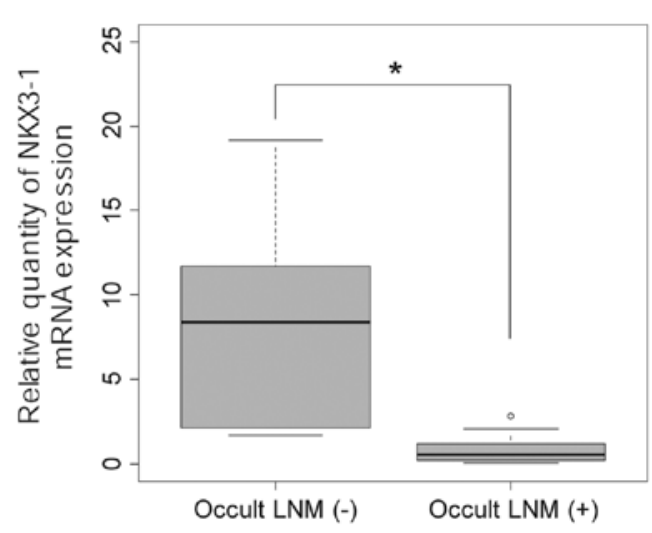

Figure 2. A box-plot for quantitative analysis of $N K X 3-1$ gene expression between 2 groups ( 9 cases without occult LNM, 10 cases with occult LNM). p-value was calculated by the Wilcoxon rank sum test. ${ }^{*} \mathrm{p}<0.001$.

org/). The Wilcoxon rank sum test was applied to determine the difference in the relative quantities of gene expression between the occult LNM positive and negative cases. In the IHC assay, the difference of the positive staining rates between the occult LNM positive and negative cases was statistically analyzed by the Wilcoxon rank sum test. The survival curves were estimated by the Kaplan-Meier method and the significances of differences between the survival curves were calculated by the log-rank test. Risk factors for DFS and OS were examined by the Cox proportional hazards analysis. A probability level $<0.05$ was used for a statistical significance.

\section{Results}

Correlation analysis between CNAs and occult LNM. We analyzed the correlation between CNA data for genes and the presence of occult LNM using the Fisher's exact test. The heatmap in Fig. 1 shows that several gene clusters of loss/ deletion or gain/amplification of genes were significantly associated with occult LNM $(\mathrm{p}<0.05)$. Through a literature survey using PubMed (http://www.ncbi.nlm.nih.gov/ pubmed/), we narrowed down the significant genes detected by the Fisher's exact test. We then focused on NKX3-1, which is one of the homeodomain-containing transcription factors, encoded on 8p21.2 and known as a putative prostate tumor suppressor gene (14-17). Therefore, we further investigated its validity as a marker in OSCC through the qRT-PCR and IHC assays. The clinical characteristics of the 60 OSCC patients and correlation with $N K X 3-1$ loss are summarized in Table I. Recurrence $(p=0.012)$ and occult LNM $(p<0.001)$ were significantly associated with loss of NKX3-1 and none of the other factors was significant.

Quantitative RT-PCR verifies gene expression for NKX3-1 and correlation with $V E G F-C$. The gene expression analysis for $N K X 3-1$ was performed by quantitative real-time RT-PCR in order to verify whether the DNA copy number of $N K X 3-1$ affected the gene expression in the 19 OSCC cases ( 9 cases without occult LNM, 10 cases with occult LNM) in the original sample set. The result of quantitative analysis 
A
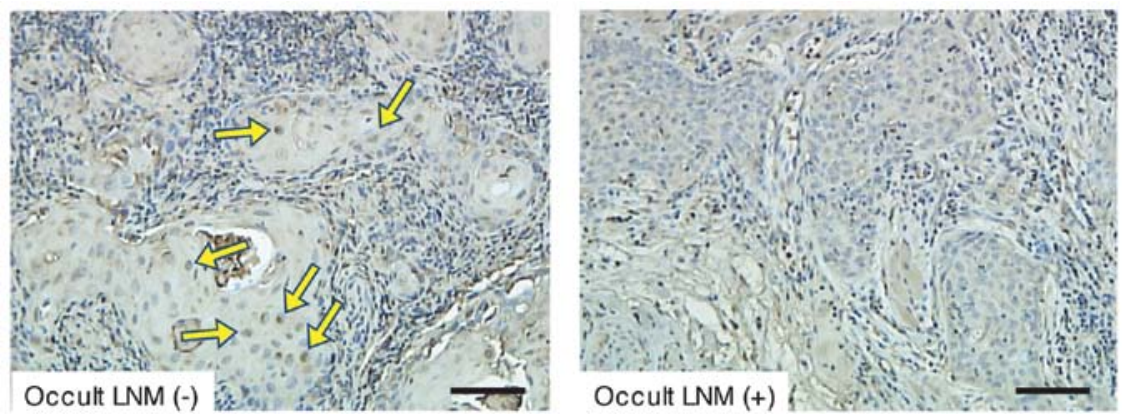

B

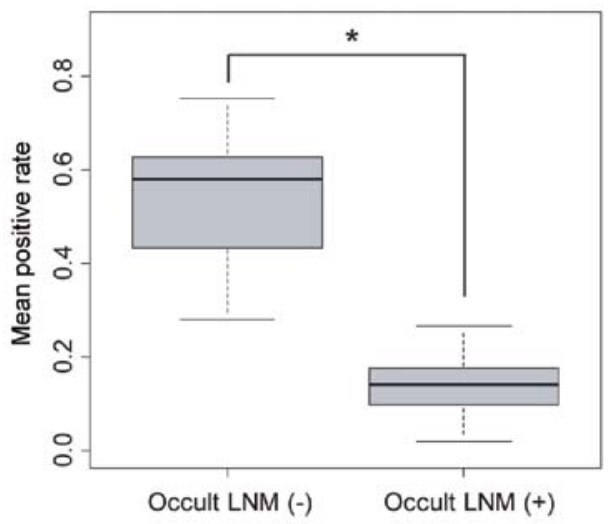

C

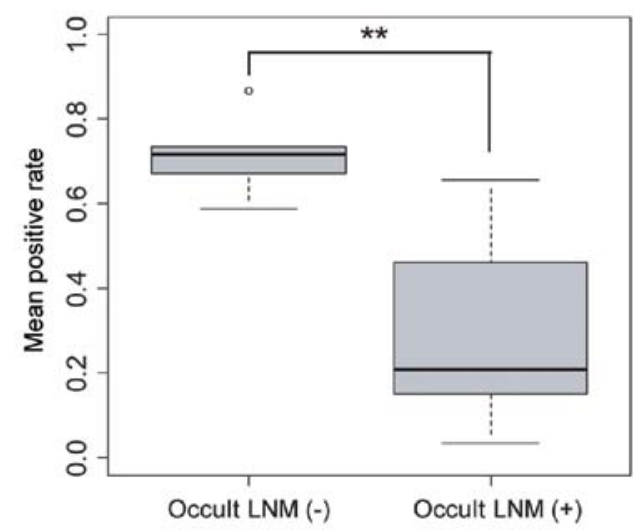

Figure 3. Immunohistochemistry for FFPE OSCC tissues with monoclonal anti-NKX3-1 antibody (1:200). (A) Positive staining (pointed by yellow arrows) for NKX3-1 was more frequently observed in the group without occult LNM than the group with occult LNM. Scale bar: $100 \mu \mathrm{m}$. (B) the Wilcoxon rank sum test was applied for the IHC results to test the difference of average positive rates between OSCC cases with and without occult LNM. ${ }^{*} \mathrm{p}<0.001$. (C) the Wilcoxon rank sum test was applied for the IHC results to test the difference of average positive rates between the independent OSCC cases with and without occult LNM. ${ }^{* *} \mathrm{p}=0.004$.

A

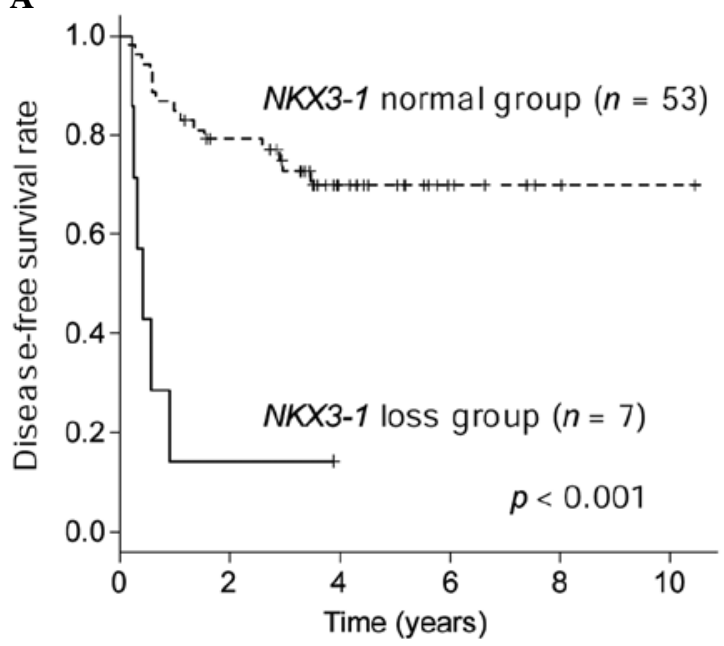

B

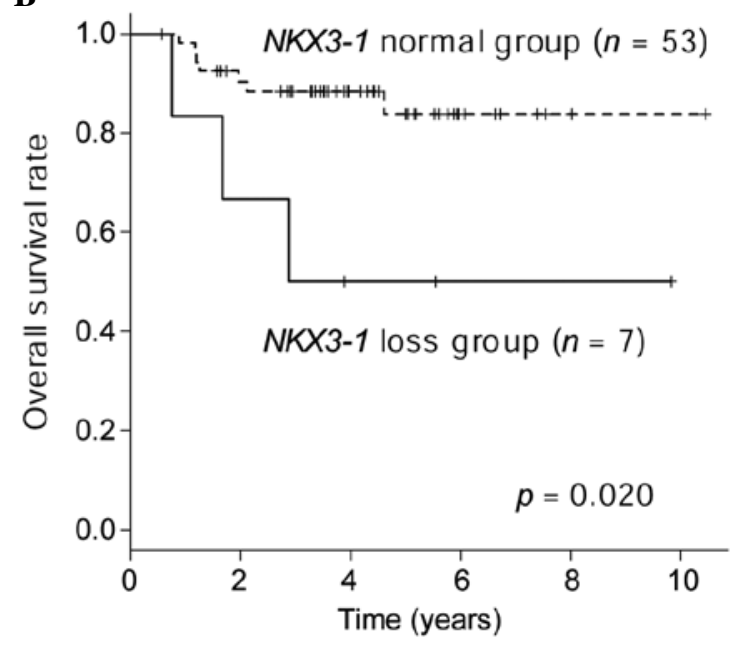

Figure 4. Disease-free and overall survival curves analyzed for the 60 OSCC cases with the Kaplan-Meier method. (A) The disease-free survival rate of patients with $N K X 3-1$ loss was significantly lower than that of patients with normal copy number of $N K X 3-1$ (log-rank test, $\mathrm{p}<0.001)$. (B) The overall survival rate of patients with $N K X 3-1$ loss was significantly lower than that of patients with normal copy number of $N K X 3-1$ (log-rank test, $\mathrm{p}=0.020)$.

showed a significant decrease $(\mathrm{p}<0.001)$ in the mean relative gene expression of $N K X 3-1$ in the OSCC cases with occult LNM (Fig. 2). To investigate the transcriptional influence of $N K X 3-1$ expression on $V E G F-C$, the quantitative results from qRT-PCR were compared. A weak negative correlation of $\mathrm{r}=-0.256$ was observed between the two genes although it did not reach a statistical significance.

Protein expression level of NKX3-1 in occult LNM cases. Following the verification of DNA copy number loss and tran- 
Table II. Cox proportional hazard analysis for clinicopathological factors with DFS and OS.

\begin{tabular}{|c|c|c|c|c|c|c|}
\hline \multirow[b]{2}{*}{ Variables } & \multicolumn{3}{|c|}{ DFS } & \multicolumn{3}{|c|}{ OS } \\
\hline & HR & $95 \% \mathrm{CI}$ & p-value & HR & $95 \% \mathrm{CI}$ & p-value \\
\hline $\begin{array}{l}\text { Age } \\
<60 \\
\geq 60\end{array}$ & 0.975 & $(0.411-2.314)$ & 0.954 & 1.848 & $(0.477-7.152)$ & 0.374 \\
\hline $\begin{array}{l}\text { Gender } \\
\text { Male } \\
\text { Female }\end{array}$ & 0.797 & $(0.336-1.893)$ & 0.608 & 0.949 & $(0.266-3.383)$ & 0.936 \\
\hline $\begin{array}{l}\text { Alcohol habit } \\
\text { Negative } \\
\text { Positive }\end{array}$ & 0.614 & $(0.238-1.585)$ & 0.313 & 0.634 & $(0.151-2.658)$ & 0.533 \\
\hline $\begin{array}{l}\text { Smoking habit } \\
\text { Negative } \\
\text { Positive }\end{array}$ & 1.797 & $(0.707-4.569)$ & 0.218 & 1.284 & $(0.321-5.140)$ & 0.724 \\
\hline $\begin{array}{l}\text { Growth pattern } \\
\text { Superficial/exophitic } \\
\text { Endophitic }\end{array}$ & 0.884 & $(0.355-2.200)$ & 0.790 & 1.587 & $(0.425-5.919)$ & 0.492 \\
\hline $\begin{array}{l}\text { Cellular differentiation } \\
\text { Well/Moderate } \\
\text { Poor }\end{array}$ & 1.004 & $(0.296-3.414)$ & 0.994 & 2.694 & $(0.694-10.460)$ & 0.152 \\
\hline $\begin{array}{l}\text { Pathological T stage } \\
\text { I, II } \\
\text { III, IV }\end{array}$ & 1.014 & $(0.371-2.769)$ & 0.978 & 1.628 & $(0.420-6.309)$ & 0.481 \\
\hline $\begin{array}{l}\mathrm{pN} \\
\text { Negative } \\
\text { Positive }\end{array}$ & 1.861 & $(0.783-4.426)$ & 0.160 & 2.560 & $(0.738-8.874)$ & 0.138 \\
\hline $\begin{array}{l}\text { Mode of invasion } \\
1-3 \\
4 C, 4 D \\
\text { Undetermined }\end{array}$ & 1.260 & $(0.464-3.418)$ & 0.650 & 2.222 & $(0.555-8.903)$ & 0.259 \\
\hline $\begin{array}{l}\mathrm{CN} \text { of } N K X 3-1 \\
\text { Normal } \\
\text { Loss }\end{array}$ & 7.574 & $(2.835-20.240)$ & $<0.001$ & 4.351 & $(1.124-16.840)$ & 0.033 \\
\hline
\end{tabular}

DFS, disease-free survival; OS, overall survival; HR, hazard ratio.

scriptional down-regulation of $N K X 3-1$, the protein expression of NKX3-1 was also determined by the IHC assay. NKX3-1, a transcriptional factor, was stained in a nucleus of oral squamous malignant cell and less stained in the occult LNM-positive cases (Fig. 3A). The average positive rates of NKX3-1 staining were significantly lower $(\mathrm{p}<0.001)$ in the group with occult LNM (Fig. 3B). The significant difference was validated by the additional IHC assay in the independent sample set and the average positive rates of NKX3-1 was significantly lower in occult LNM-positive cases ( $\mathrm{p}=0.004$; Fig. $3 \mathrm{C}$ ).

Loss of NKX3-1 is correlated with low survival rates. To determine the correlation between loss of $N K X 3-1$ and survival rates in the OSCC patients $(n=60)$, the Kaplan-Meier curves were constructed for both DFS and OS. Among the OSCC patients, the cases with loss in $N K X 3-1$ had a significantly lower DFS rate (log-rank test, $\mathrm{p}<0.001$; Fig. $4 \mathrm{~A}$ ). The OS analysis also demonstrated a significant difference between the patients with and without loss of $N K X 3-1$ (log-rank test, $p=0.020$; Fig. 4B). Among the various clinicopathological parameters, the Cox proportional hazards analysis showed that loss of $N K X 3-1$ was the only factor significantly associated with poor prognosis (Table II).

\section{Discussion}

Precedent studies demonstrated that genome-wide CNA analyses could identify CNA-based markers associated with metastasis in breast cancer, colorectal cancer and endometrioid carcinoma (18-20). Therefore, a comprehensive CNA 
analysis with microarray can be valuable in an investigation of causative genomic changes for occult LNM of OSCC. To the best of our knowledge, this is the first study of genome-wide aberrations of DNA copy number using Mapping 250K array on primary tumor tissues as metastatic profiles after surgical treatment of primary OSCC sites.

The present study identified a significant correlation between loss of NKX3-1 and occult LNM of OSCC based on the genome-wide CNA analysis on the 60 OSCC patients. The results of quantitative RT-PCR and IHC revealed significantly lower expression of NKX3-1 in the primary tumor of the occult LNM cases. The Kaplan-Meier analysis showed that both DFS and OS rates significantly decreased in the patients with NKX3-1 loss. The expression pattern of NKX3-1 protein was reproduced in the independent patient group, i.e., the expression in the patients with occult LNM was significantly lower than those without occult LNM. All these findings indicated that loss of $N K X 3-1$ is involved in occult LNM in OSCC. This is the first report that loss of $N K X 3-1$ is significantly associated with the presence of occult LNM in OSCC although there are several papers reporting involvement of NKX3-1 mostly in prostate cancer (14-17,21-23) but a few in acute lymphoblastic leukemia (24), adrenal gland tumor (25), breast cancer $(26,27)$, kidney cancer $(25,28)$ and testis cancer $(25,29)$. In the following, we will discuss possible roles of NKX3-1 in occult LNM of OSCC and its validity as a marker.

Chromosomal site $8 \mathrm{p}$, where NKX3-1 gene is encoded, is frequently reported as one of the major alteration sites having genomic loss and LOH which are associated with tumorigenesis, metastasis or poor prognosis of cancer patients. It has been discussed that some of the genes encoded on the region might be tumor suppressor genes and potential markers for tumorigenesis, metastasis or poor prognosis (30-33). NKX3-1, which is one of the homeodomain-containing transcriptional factors, was first reported for an association with prostate cancer in 1996 (15). From that time on, NKX3-1 has been studied for the involvement in the carcinogenesis and progression of prostate cancer as a putative prostate tumor suppressor gene that shows prostate-specific gene expression $(16,17,22)$. Some reports showed that in prostate cancer the DNA copy number loss or down-regulation of $N K X 3-1$ had a significant correlation with progression, LNM and poor prognosis $(14,21,23)$. In breast cancer, $N K X 3-1$ was considered as a potential transcriptional inhibitor of estrogen receptor response and tumor suppressor gene $(26,27)$. NKX3-1 was also identified in prostate and kidney cancer cell lines as an enhancer of topoisomerase I, which is an enzyme regulating the overwinding or underwinding of DNA and involved in DNA replication, transcription, or DNA repair (28). On the other hand NKX3-1 could have an activity of either tumor suppressor or oncogene in $\mathrm{T}$ cell acute lymphoblastic leukemia depending on tissue expression (24).

Interestingly, NKX3-1 has a binding domain to the promoter region of $V E G F-C$, which is a lymphangiogenesis factor, and NKX3-1 normally regulates the expression of $V E G F-C$ (13). Lymphangiogenesis is the formation of novel lymphatic vessels from existing lymphatic vessels. Significant involvement of VEGF family in vasculogenesis, angiogenesis and lymphangiogenesis has been reported (34). Tumor cells require angiogenesis to obtain oxygen and nutrients from a regular blood stream, and overexpressions of VEGF proteins are observed in several malignancies $(35,36)$. In addition, progression and metastasis of tumor cells and poor prognosis are also correlated with overexpressions of VEGFs (37-40). VEGF-C is classified into lymphatic markers, which are desmoplakin, LYVE-1, PROX-1, VEGF-C, VEGF-D and VEGFR-3, and generally studied in the correlation with nodal metastasis of cancers $(36,41)$. Therefore, up-regulation of VEGF-C is induced by reduction of NKX3-1 expression, resulting in driving lymphangiogenesis (13).

Based on these facts, we hypothesize that lymphangiogenesis induced by VEGF-C is suppressed under the normal condition of cells because NKX3-1 negatively regulates transcription of $V E G F-C$. By contrast, novel lymphatic vessels may tend to be produced around tumor cells while up-regulation of VEGF-C is induced by reduction of NKX3-1 expression. In consequence, metastatic tumor cells can spread to regional lymph nodes through the newly produced and existing lymphatic vessels, followed by extravasation and proliferation in lymph nodes, resulting in the metastasis in regional lymph nodes. Since status of occult LNM is one of the critical risk factors to lower the OS rate, the significant decrease in OS rate observed in the OSCC patients with loss of $N K X 3-1$ can be explained in part by $N K X 3-1$ loss being a risk factor to increase an incidence of occult LNM. Amplifications of CCND1 and CCNL1 have been reported as CNA-based promising biomarkers for occult LNM $(11,12)$. Using the new marker, loss of $N X K 3-1$, in addition could improve the accuracy of prediction for occult LNM. In this study, although we focused only on NKX3-1, we will continue to investigate the other genes significantly associated with occult LMN as potential biomarkers.

In conclusion, the genome-wide CNA analyses on 60 OSCC patients identified several genes having significant correlation with occult LNM. We then focused on the significant correlation between loss of $N K X 3-1$ and occult LNM based on the literature. Our findings suggest that DNA copy number loss and low expression of $N K X 3-1$ may be one of the responsible factors for driving the lymphangiogenesis to accomplish metastasis at the secondary site, implying that NKX3-1 becomes a novel prognostic factor and a promising biomarker to improve a molecular-based prediction of occult LNM in patients with OSCC. Together with the conventional biomarkers, loss of NKX3-1 would provide insight into selecting an appropriate management approach.

\section{Acknowledgements}

We thank World Fusion Co., Ltd., especially Mr. R. Sato and Mr. K. Kawahara, for helping with the development of the CNA analytical tool. This work was supported in part by grant-inaids from Science and Technology Promotion Adjustment Expenses of Japan (no. 08005234) and Integrated Database Project, Ministry of Education, Culture, Sports, Science and Technology in Japan.

\section{References}

1. Jemal A, Bray F, Center MM, Ferlay J, Ward E and Forman D: Global cancer statistics. CA Cancer J Clin 61: 69-90, 2011. 
2. Matsuda T, Marugame T, Kamo K, Katanoda K, Ajiki W and Sobue T: Cancer incidence and incidence rates in Japan in 2005: based on data from 12 population-based cancer registries in the Monitoring of Cancer Incidence in Japan (MCIJ) project. Jpn J Clin Oncol 41: 139-147, 2011

3. Tankere F, Camproux A, Barry B, Guedon C, Depondt J and Gehanno P: Prognostic value of lymph node involvement in oral cancers: a study of 137 cases. Laryngoscope 110: 2061-2065, 2000.

4. Haddadin KJ, Soutar DS, Oliver RJ, Webster MH, Robertson AG and MacDonald DG: Improved survival for patients with clinically T1/T2, N0 tongue tumors undergoing a prophylactic neck dissection. Head Neck 21: 517-525, 1999.

5. Yuen AP, Wei WI, Wong YM and Tang KC: Elective neck dissection versus observation in the treatment of early oral tongue carcinoma. Head Neck 19: 583-588, 1997.

6. Weiss MH, Harrison LB and Isaacs RS: Use of decision analysis in planning a management strategy for the stage N0 neck. Arch Otolaryngol Head Neck Surg 120: 699-702, 1994.

7. Yaginuma Y, Unotoro J, Kamiyama H, et al: Genomic copynumber aberrations related to lymph-node metastasis of colon cancer. J Int Med Res 34: 390-396, 2006.

8. Yamamoto S, Midorikawa Y, Morikawa T, et al: Identification of chromosomal aberrations of metastatic potential in colorectal carcinoma. Genes Chromosomes Cancer 49: 487-496, 2010.

9. Nakaya K, Yamagata HD, Arita N, et al: Identification of homozygous deletions of tumor suppressor gene FAT in oral cancer using CGH-array. Oncogene 26: 5300-5308, 2007.

10. Baldwin C, Garnis C, Zhang L, Rosin MP and Lam WL: Multiple microalterations detected at high frequency in oral cancer. Cancer Res 65: 7561-7567, 2005.

11. Sticht C, Hofele C, Flechtenmacher C, et al: Amplification of Cyclin L1 is associated with lymph node metastases in head and neck squamous cell carcinoma (HNSCC). Br J Cancer 92: 770-774, 2005

12. Myo K, Uzawa N, Miyamoto R, Sonoda I, Yuki Y and Amagasa T: Cyclin D1 gene numerical aberration is a predictive marker for occult cervical lymph node metastasis in TNM Stage I and II squamous cell carcinoma of the oral cavity. Cancer 104: 2709-2716, 2005.

13. Zhang H, Muders MH, Li J, Rinaldo F, Tindall DJ and Datta K: Loss of NKX3.1 favors vascular endothelial growth factor-C expression in prostate cancer. Cancer Res 68: 8770-8778, 2008.

14. Asatiani E, Huang WX, Wang A, et al: Deletion, methylation, and expression of the NKX3.1 suppressor gene in primary human prostate cancer. Cancer Res 65: 1164-1173, 2005.

15. Bieberich CJ, Fujita K, He WW and Jay G: Prostate-specific and androgen-dependent expression of a novel homeobox gene. J Biol Chem 271: 31779-31782, 1996.

16. He WW, Sciavolino PJ, Wing J, et al: A novel human prostatespecific, androgen-regulated homeobox gene (NKX3.1) that maps to $8 \mathrm{p} 21$, a region frequently deleted in prostate cancer Genomics 43: 69-77, 1997.

17. Prescott JL, Blok L and Tindall DJ: Isolation and androgen regulation of the human homeobox cDNA, NKX3.1. Prostate 35 71-80, 1998.

18. Zhang Y, Martens JW, Yu JX, et al: Copy number alterations that predict metastatic capability of human breast cancer. Cancer Res 69: 3795-3801, 2009.

19. Suehiro Y, Umayahara K, Ogata H, et al: Genetic aberrations detected by comparative genomic hybridization predict outcome in patients with endometrioid carcinoma. Genes Chromosomes Cancer 29: 75-82, 2000

20. Tanami H, Tsuda H, Okabe S, et al: Involvement of cyclin D3 in liver metastasis of colorectal cancer, revealed by genome-wide copy-number analysis. Lab Invest 85: 1118-1129, 2005.

21. Abate-Shen C, Banach-Petrosky WA, Sun X, et al: Nkx3.1; Pten mutant mice develop invasive prostate adenocarcinoma and lymph node metastases. Cancer Res 63: 3886-3890, 2003.
22. Bhatia-Gaur R, Donjacour AA, Sciavolino PJ, et al: Roles for Nkx3.1 in prostate development and cancer. Genes Dev 13: 966-977, 1999.

23. Bowen C, Bubendorf L, Voeller HJ, et al: Loss of NKX3.1 expression in human prostate cancers correlates with tumor progression. Cancer Res 60: 6111-6115, 2000

24. Kusy S, Gerby B, Goardon N, et al: NKX3.1 is a direct TAL1 target gene that mediates proliferation of TAL1-expressing human T cell acute lymphoblastic leukemia. J Exp Med 207: 2141-2156, 2010

25. Srivastava M, Torosyan Y, Raffeld M, Eidelman O, Pollard HB and Bubendorf L: ANXA7 expression represents hormonerelevant tumor suppression in different cancers. Int J Cancer 121: 2628-2636, 2007.

26. Lin H, Xiao J, Luo X, et al: Overexpression HERG K(+) channel gene mediates cell-growth signals on activation of oncoproteins SP1 and NF-kappaB and inactivation of tumor suppressor Nkx3.1. J Cell Physiol 212: 137-147, 2007.

27. Holmes KA, Song JS, Liu XS, Brown M and Carroll JS: Nkx3-1 and LEF-1 function as transcriptional inhibitors of estrogen receptor activity. Cancer Res 68: 7380-7385, 2008

28. Bowen C, Stuart A, Ju JH, et al: NKX3.1 homeodomain protein binds to topoisomerase I and enhances its activity. Cancer Res 67: 455-464, 2007.

29. Skotheim RI, Korkmaz KS, Klokk TI, et al: NKX3.1 expression is lost in testicular germ cell tumors. Am J Pathol 163: 2149-2154, 2003.

30. Kujawski M, Sarlomo-Rikala M, Gabriel A, Szyfter K and Knuutila S: Recurrent DNA copy number losses associated with metastasis of larynx carcinoma. Genes Chromosomes Cancer 26: 253-257, 1999.

31. Zhou X, Jordan RC, Li Y, Huang BL and Wong DT: Frequent allelic imbalances at $8 p$ and $11 q 22$ in oral and oropharyngeal epithelial dysplastic lesions. Cancer Genet Cytogenet 161: 86-89, 2005.

32. Ye H, Pungpravat N, Huang BL, et al: Genomic assessments of the frequent loss of heterozygosity region on 8p21.3-p22 in head and neck squamous cell carcinoma. Cancer Genet Cytogenet 176: 100-106, 2007.

33. Yoo WJ, Cho SH, Lee YS, et al: Loss of heterozygosity on chromosomes $3 p, 8 p, 9 p$ and $17 p$ in the progression of squamous cell carcinoma of the larynx. J Korean Med Sci 19: 345-351, 2004.

34. Koch S, Tugues S, Li X, Gualandi L and Claesson-Welsh L: Signal transduction by vascular endothelial growth factor receptors. Biochem J 437: 169-183, 2011.

35. Carmeliet P and Jain RK: Principles and mechanisms of vessel normalization for cancer and other angiogenic diseases. Nat Rev Drug Discov 10: 417-427, 2011.

36. Carmeliet $P$ and Jain RK: Molecular mechanisms and clinical applications of angiogenesis. Nature 473: 298-307, 2011.

37. Kishimoto K, Sasaki A, Yoshihama Y, Mese H, Tsukamoto G and Matsumura T: Expression of vascular endothelial growth factor-C predicts regional lymph node metastasis in early oral squamous cell carcinoma. Oral Oncol 39: 391-396, 2003.

38. Uehara M, Sano K, Ikeda $\mathrm{H}$, et al: Expression of vascular endothelial growth factor and prognosis of oral squamous cell carcinoma. Oral Oncol 40: 321-325, 2004.

39. Siriwardena BS, Kudo Y, Ogawa I, Udagama MN, Tilakaratne WM and Takata T: VEGF-C is associated with lymphatic status and invasion in oral cancer. J Clin Pathol 61: 103-108, 2008.

40. Shintani S, Li C, Ishikawa T, Mihara M, Nakashiro K and Hamakawa $\mathrm{H}$ : Expression of vascular endothelial growth factor A, B, C, and D in oral squamous cell carcinoma. Oral Oncol 40: 13-20, 2004.

41. Liu X, Kolokythas A, Wang J, Huang H and Zhou X: Gene expression signatures of lymph node metastasis in oral cancer: Molecular Characteristics and Clinical Significances. Curr Cancer Ther Rev 6: 294-307, 2010. 